\title{
LA PRESENCIA BOLIVIANA EN LA SOCIEDAD DEL SALITRE Y LA NUEVA DEFINICIÓN DE LA FRONTERA: AUGE Y CAÍDA DE UNA DINÁMICA TRANSFRONTERIZA (TARAPACÁ 1880-1930)
}

\author{
THE BOLIVIAN PRESENCE IN THE NITRATE SOCIETY AND THE NEW \\ DEFINITION OF THE FRONTIER: THE RISE AND FALL OF A TRANSBORDER \\ DYNAMIC (TARAPACÁ 1880-1930)
}

Sergio González, Miranda ${ }^{1,2}$

\begin{abstract}
El presente artículo analiza la presencia de población boliviana durante el ciclo de expansión del nitrato (1880-1930) en la tradicional provincia de Tarapacá, abordando las dinámicas transfronterizas de trabajadores y bienes, en particular el fenómeno del enganche salitrero. El paso de la frontera peruano-boliviana a chileno-boliviana.

La hipótesis de este trabajo plantea que la presencia boliviana en la industria del nitrato está relacionada con las relaciones diplomáticas entre ambos países, por un lado, y en la atracción del enclave salitrero, por otro. Se hace una comparación introductoria entre la frontera chileno-peruana y la frontera chileno-boliviana, señalando que la primera tuvo una frontera cerrada debido al impacto de las conflictivas relaciones diplomáticas entre Perú y Chile, mientras la segunda fue una frontera abierta, debido a los intereses empresariales salitreros y una coincidencia en el modelo exportador entre los gobiernos de Bolivia y Chile, que posibilitó el acuerdo diplomático expresado en el tratado de 1904.

El enfoque de este estudio se mueve desde las relaciones internacionales, específicamente las relaciones transfronterizas, hasta la historia de la vida privada de las poblaciones bolivianas en la sociedad del salitre, demostrando la complejidad del problema estudiado.
\end{abstract}

Palabras claves: relaciones diplomáticas, frontera, industria del salitre, migraciones.

The current article analyzes the presence of Bolivians in the expansion cycle of nitrate (1880-1930) in the traditional province of Tarapacá, tackling the transborder dynamics of workers and goods, in particular the phenomenon of saltpeter refinery recruitment. Change from a Peruvian-Bolivian border to a Chilean-Bolivian border.

The hypothesis of this paper proposes that the Bolivian presence in the nitrate industry is related to diplomatic relations between both countries, on the one hand, and on the attraction of the saltpeter refinery enclave on the other. A preliminary comparison is made between the Chilean-Peruvian frontier and the Chilean-Bolivian frontier, pointing out that former had a closed frontier due to the impact of the controversial diplomatic relations between Peru and Chile, while the latter was an open frontier, due to the saltpeter refinery business interests and a coincidence in the exporting model between the governments of Bolivia and Chile, that made possible the diplomatic agreement expressed in the treaty of 1904.

The focus of this study moves from international relations, specifically transborder relations, to the history of private life of the Bolivian population in the saltpeter society, showing the complexity of the problem under study.

Key words: Diplomatic relations, frontier, saltpeter industry, migrations.

Puede resultar paradójico que, una vez concluida la guerra del salitre (1879-1883), cuando las provincias bolivianas del Litoral (Toco y Antofagasta) y las peruanas de Tarapacá, Arica y Tacna fueron anexadas a Chile (temporal o definitivamente), las dinámicas de las fronteras en estas nuevas provincias chilenas fueran tan diferentes. Mientras la frontera entre Perú y Chile inicia un largo y enojoso camino de conflictos diplomáticos y sociales, la frontera entre Bolivia y Chile goza de un proceso de integración física, económica y poblacional que no volverá a conocerse después de concluido el ciclo de expansión del nitrato, aunque no sin dificultades.

El ciclo de expansión del nitrato, que marcó la economía chilena, se extendió aproximadamente entre 1880 y 1930, medio siglo donde esta actividad productiva se ubicó en el desierto de Atacama entre Pisagua y Taltal. Aquí se analiza solamente lo ocurrido en la provincia de Tarapacá, en el marco de dos preguntas: ¿Cuál fue la importancia de la presencia boliviana en la sociedad y economía salitreras?; y ¿cómo se definió la frontera en esa época entre Bolivia y Chile?

\footnotetext{
1 Centro de Investigaciones del Hombre en el Desierto (CIHDE), Iquique, Chile.
}

2 Universidad Arturo Prat, Iquique, Chile. sgonzale@unap.cl 
Conocida es la presencia de población peruana y boliviana en todo el norte salitrero, de manera especial en Tarapacá, debido al origen peruano de esta provincia y a la proximidad con el altiplano boliviano. Observaremos la importancia de ambas nacionalidades en las faenas del nitrato, donde la presencia boliviana irá consolidándose mientras la peruana comenzará a perder importancia en la medida que el ciclo se desarrollaba.

No profundizaremos la problemática fronteriza entre Perú y Chile, diremos solamente que dicho conflicto se prolongó desde 1884 hasta 1929, debido a la decisión política de dejar pendiente la soberanía de las provincias de Tacna y Arica, ocupadas durante todo ese período por Chile y protestadas por Perú. Hacia 1894, año en el que debió realizarse el plebiscito por las provincias en comento, según lo establecía el Tratado de Ancón, no se llevó a cabo este acto cívico debido a la inexistencia de un protocolo o reglamento que estableciera las características de la consulta, y porque Perú no estaba en condiciones económicas para pagar la indemnización a Chile si ganaba el plebiscito. La no realización de esta consulta fue la base de la impugnación peruana al Tratado de Ancón y la fuente del conflicto en las décadas siguientes. Autores peruanos (p.ej. Palacios 1974) coinciden en que el fracaso del protocolo Billinghurst-Latorre hacia 1901 marcó el inicio de un conflicto no sólo diplomático sino social y político, que sólo concluirá con el Tratado de Lima de 1929.

En cambio, en Tarapacá, provincia anexada de forma definitiva y perpetua, según el artículo segundo del Tratado de Ancón de octubre de $1883^{1}$, podría esperarse que sus límites fueran claros y precisos. Uno, porque ya era una provincia peruana consolidada $\mathrm{y}$, por lo mismo, supuestamente con fronteras bien delimitadas con Bolivia, tanto al este como al sur. Dos, porque su límite interno con la provincia de Arica estaba bien marcado por la profunda quebrada de Camarones. Tres, porque además del Tratado de Ancón con Perú, Chile había firmado un Pacto de Tregua con Bolivia el 4 de abril de 1884, lo que daba supuestamente tranquilidad en esas fronteras internacionales.

Si bien comparativamente con la frontera norte, en Tacna y Arica, Chile enfrentó en Tarapacá menos problemas fronterizos, no por ello estuvo ajeno a dificultades de sus límites. En términos generales, se puede afirmar que Chile tuvo fronteras con Bolivia expeditas y seguras para el tránsito de personas (como fue el caso de los enganches ${ }^{2}$ a las oficinas salitreras) y de bienes (como fue el caso del intercambio de productos andinos con aquellos llegados al litoral a través del cabotaje), pero ello no significó que estuvo exento de litigios tanto en localidades bien determinadas como en amplias zonas.

Un ejemplo de la importancia de esta escasa definición de los límites internacionales entre Perú y Bolivia en las provincias anexadas por Chile, lo tenemos en el siguiente párrafo del perito chileno Luis Riso Patrón:

Según el legajo de copias (doc. Núm. 75), remitidas por nuestra Legación en La Paz, i el Memorándum firmado por el Jefe de la Oficina de Límites doctor Bautista Saavedra (doc. Núm. 7) Bolivia podría legalmente pretender en la provincia de Carangas a la posesión de una ancha faja de la provincia de Tacna i a la quebrada de Camarones casi hasta la del meridiano de Codpa, incluyendo los pueblos de Esquiña i Pachica.

Al tratar del límite oriental de la provincia de Tacna, he dejado bien establecido que los pretendidos derechos de Bolivia allí han sido siempre letra muerta.

Según el Memorándum aludido, la línea boliviana debería pasar por "el pueblo de Guallatiri; de aquí a Anocarire, a Chulluncani, a Surira, a Molluri, a Pumire; de Pumire debería pasar la línea por Pasirigo, Apacheta de Parinacota, cerro de Latarine, Cala Cruz, Chillacollo, Chiarvito, Caraguano a Chiapi-apacheta. No se debe incluir Rinconada i Hanco-Apacheta que están actualmente en posesión boliviana, i la Rinconada hasta Hanco-Apacheta. Verdad que dentro de la última parte del deslinde a partir de Cala Cruz, quedaría el pueblo de Isluga a favor de Bolivia; pero si no es posible esto, entonces la línea de demarcación seguirá de Cala Cruz al volcán Isluga con rumbo S. O. a N.E”.

Los términos mismos en que está espresada la pretensión la desvirtúan, pues está en contradicción con los documentos en que pretende fundarse, como ya se ha visto respecto de Tacna (Riso Patrón 1910:72).

Si observamos la actual frontera entre Chile y Bolivia en los puntos señalados podemos concluir 
que se trata de grandes extensiones de territorios, que se ubican por casi toda la frontera de las actuales provincias de Arica y Tarapacá, demostrando con ello la importancia de los peritos, quienes debieron recurrir a las más diversas fuentes para consolidar sus posiciones en beneficio de sus respectivos países.

No analizaremos aquí la frontera sur de la provincia de Tarapacá, porque en rigor en este punto esta provincia ya no limitaba con Bolivia, sino con la provincia de Antofagasta ocupada por Chile. Ese límite estaba definido por "la quebrada y el río Loa hasta el pueblo de Quillagua inclusive, y desde ese punto una línea que, tocando en los volcanes Miño, Olca y volcán Túa, llegue hasta la frontera boliviana" (Riso Patrón 1910:5). Esta frontera sur y la frontera norte (quebrada de Camarones) ${ }^{3}$ no estuvieron exentas de problemas y litigios antes de los Tratados de 1904 con Bolivia y de 1929 con Perú, pero no corresponden ser analizados aquí. Analizaremos, en cambio, la frontera este de Tarapacá, desde donde llegaron los principales flujos de bienes y personas hacia las salitreras y, de igual modo, desde donde éstas retornaron hacia Bolivia, generando una dinámica transfronteriza de gran densidad, que sólo decayó con la gran crisis del nitrato en la década de $1930^{4}$.

\section{La Frontera de Tarapacá con Bolivia}

Esta frontera estaba lejos de estar bien definida por Perú y Bolivia hacia 1879, quedando entre esta fecha y el Tratado de 1904 en un estado de apertura que, en cierto modo, fue funcional a la dinámica requerida por la sociedad y economía del salitre. Por lo mismo, con excepción de las aduanillas de Oruro, no existen registros de los flujos de bienes y personas que circularon por esa frontera en el período estudiado, con excepción del relato oral.

Peritos chilenos como Alejandro Bertrand y Luis Riso Patrón, entre otros, debieron basarse en geógrafos y diplomáticos peruanos y extranjeros, como Mariano Paz Soldán, Hugo Reck o Guillermo Billinghurst, para ir determinando la línea de la frontera con Bolivia en el tramo correspondiente a Tarapacá, que por cierto no estuvo ajena al conflicto. Luis Riso Patrón señala:

Respecto a la frontera pretendida por Bolivia en su provincia de Carangas con Tarapacá, es decir al sur de Camarones, no aparece mas justificada. El documento en que la funda el doctor Saavedra es encabezado por una petición del cacique Ildefonso Casiguaman del pueblo de Sabaya "que se queja de invasiones de los del pueblo de Isluga de la jurisdicción de Arica, sobre los de paso de Pisiga que están una legua mas al oriente del mojón de deslinde" (Memorándum, doc. Núm. 74 p. 9).

Hai entre esta aserción i la pretendida demarcación que se establece en seguida una contradicción i aun dos, que desautorizan completamente el documento que la contiene, i son las siguientes:

Si los del pueblo de Isluga, de la jurisdicción de Arica, hacían invasiones en la de Caranguas, ¿cómo podía dicho pueblo quedar incluido en esta última, según resultaría la demarcación pretendida ahora por Bolivia? Asimismo, si la ciénega de Pisiga estaba sólo una legua al oriente del mojón de deslinde (mojón cerro Prieto, nombrado en las actas de amojonamiento) ¿cómo se pretende que en esa época pasaba el deslinde Pasirijo i el cerro de Latarani que están muchas leguas al poniente de dicha ciénega como puede verse en el mapa? (Riso Patrón 1910:73).

Lo interesante es observar que el comportamiento histórico de los comuneros fue parte del argumento en la definición de la frontera, es decir, la dinámica transfronteriza se constituyó en prueba de soberanía. Un argumento esencial fue el lugar donde los comuneros habían inscrito sus tierras, porque si lo hicieron en el puerto de Pisagua era un indicador claro del dominio soberano peruano. Por lo mismo, los peritos debieron recurrir al testimonio oral de lugareños que por usos y costumbres tenían mayor claridad sobre la nacionalidad de determinadas localidades. Observemos, al respecto, un interesante ejemplo del mismo perito chileno Luis Riso Patrón:

En efecto, en el curso del sumario judicial levantado con motivo de un litigio pendiente sobre pertenencias de borato de cal, han sido llamados a declarar numerosos indíjenas de Surire, Mullurem, Camiña, Isluga, etc., algunos de ellos mui ancianos, quienes estuvieron contestes en que el dominio 
peruano ántes del de Chile se estendia a todos los pastales de Surire, Chilcaya i otros vecinos.

Severo Choque, de Camiña, declara (doc. Núm. 66, páj. 12) que "en 1879 estuvo en Chilcaya pasteando ganados, i siempre dependiente de las autoridades de Camiña... agrega que hace mas de veinte años se promovieron pleitos entre los vivientes de Surire i que el teniente gobernador de Mullure, señor Anjel Custodio Fernández, acompañado del comisionado señor José M. Araníbar nombrado por el prefecto de Tarapacá i de acuerdo con las autoridades de Tacna o Arica, procedieron a deslindar dichos territorios... i se fijó la siguiente línea que ha sido respetada hasta hoi por las autoridades de Arica. A partir de Canta a Pocupucuni, Llaretapampa, Chilisaga, Terrajama, Castilluma, Achechamayo i Chilcaya hasta la frontera de Bolivia al Naciente".

Don Juan B. Ossio, oriundo de Zapiga en Tarapacá "agrega que en los cerros de Arintica i Puquintica que están al costado Norte de la laguna hai gran cantidad de pedimentos i pertenencias de azufre ante el juzgado de Pisagua; algunas hai en activo trabajo habiendo pagado desde hace años sus contribuciones en Pisagua".

Posteriormente el injeniero de esta oficina don Víctor Caro Tagle, comisionado para estudiar el límite entre los departamentos de Pisagua i Arica, recorrió personalmente las localidades, guiado por un indíjena de Isluga llamado Dionisio Mamani, el cual corroboró totalmente las declaraciones mencionadas (doc. Núm. 67), en el sentido de que desde tiempo inmemorial los pastales de Chilcaya se han considerado como propiedad de la comunidad de los Islugas (Riso Patrón 1910:70-71).

La línea fronteriza entonces debió definirse entre Bolivia y Chile a partir del Tratado de 1904, donde la identidad de los lugareños para con las autoridades de uno u otro país tuvo un papel relevante. Hasta ahora no se ha reconocido la importancia de la sociedad del salitre, con su atracción hacia las comunidades altoandinas, en la integración de los comuneros que habitaban en la frontera para decidirse en apoyar a Chile y no a Bolivia en sus respectivas demandas, especialmente el papel que desempeñaron las comunidades de Isluga y Cariquima, los primeros respecto de Sabaya y los segundos con relación a Llica, los focos urbanos de atracción boliviana más próximos. La frontera definitiva no fue sinónimo de claridad y menos de resolución de conflictos. Veamos un ejemplo:

\section{REPÚBLICA DE CHILE OFICINA DE LÍMITES}

EL INFRASCRITO INGENIERO DE LA COMISIÓN DE LIMITES CON BOLIVIA HACE CONSTAR QUE ENTIENDE EN VIRTUD DEL TRATADO DE PAZ I AMISTAD CELEBRADO EL 20 DE MARZO DE 1906 ENTRE CHILE I BOLIVIA PASCUAL ESTEVAN, SANTOS ESTEVAN, SILVERIO ESTEVAN, TIBURCIO TICUNA, CONTINÚAN SIENDO DUEÑOS ABSOLUTOS DE LOS POSESIONES QUE POSEEN EN EL PUNTO LLAMADO HUAILLA SITUADO EN LA ORILLA DEL SALAR DE COIPASA AL PONIENTE DEL CERRO LACATAYA OBSTANTE QUE AL EFECTUAR LA DEMARCACIÓN DE LA LÍNEA FRONTERIZA EN ESTA REJIÓN QUE VA EN LÍNEA RECTA DESDE EL CERRITO TAPACALLO AL CERRITO SALITRAL I DE AQUÍ TAMBIÉN EN LINEAS RECTAS AL CERRO DE LACATAYA I AL PORTOSUELO DE HUAILLA ESAS POSESIONES HAYAN QUEDADO EN TERRITORIO BOLIVIANO.

HUAILLA JUNIO 14 DE 1906.

LUIS A. BOLADOS R.

(Archivo Intendencia de Tarapacá, Telegramas).

Conocemos el largo litigio que la familia Estevan llevó adelante respecto de sus propiedades, que si bien quedaron resguardadas en el acuerdo diplomático entre Bolivia y Chile, en los hechos ello no se respetó, llevándolos incluso a realizar incursiones en el territorio boliviano ${ }^{5}$. Una vez establecida una frontera internacional que irrumpe en medio de una posesión particular, los conflictos no esperan, incluso entre familiares que quedan también a veces a un lado u otro de dicha frontera. Ejemplos de ello tenemos en los pastizales de Isluga, entre los pueblos de Pisiga Carpa (chileno) y Pisiga Bolívar 
(boliviano). En la frontera peruano-chilena actual un caso notorio es el de la comunidad de Ancomarca, donde parte de ella quedó en Perú (la más importante, pues incluye el pueblo) y otra en Chile.

Mientras los peritos chilenos y bolivianos enfrentaban estas tareas para dejar bien establecida la frontera internacional, se desarrollaba en ella una dinámica generada por la atracción del enclave salitrero en el desierto de Tarapacá que, posiblemente, obligó a los geógrafos y diplomáticos a enfocar de un modo diferente esta frontera en contradicción a la geopolítica decimonónica, es decir, más proclives al acuerdo y al argumento. Si bien existen grandes diferencias entre Tarapacá y Antofagasta, el enclave salitrero en esta última provincia también tuvo un papel relevante en la definición de esas fronteras (González 2006), quizás no tanto por conflictos de comuneros como por los intereses involucrados en la explotación del nitrato, incluyendo el ferrocarril Oruro-Antofagasta que fue funcional en la movilidad de mano de obra a las salitreras. Según Harold Blakemore:

el decenio entre 1904, y el estallido de la Primera Guerra Mundial en 1914 fue el más provechoso de la Compañía (FCAB). Esto se debió sobre todo a la expansión de la economía chilena en ese período y, en especial, al crecimiento impresionante de la industria salitrera (Blakemore 1996:70).

Precisamente en esa misma década se construyó el ferrocarril de Arica a La Paz, como parte de la negociación del Tratado de Paz y Amistad entre Bolivia y Chile de 1904. Tampoco la frontera de Tarapacá ha estado ajena a la necesidad de construcción de un ferrocarril (entre Huara y Pisiga), como lo ha demostrado Luis Castro (1995). Sin embargo, los intereses salitreros y los afanes modernizadores del Estado chileno, expresados en proyectos ferroviarios, entre otros, estuvieron distantes de la población andina regional en esa época (Castro 2008). Por lo mismo, los acuerdos internacionales eran también ajenos a la población que habitaba las fronteras entre Bolivia y Chile.

La población fronteriza boliviano-chilena fue vista como mano de obra y no como una comunidad opinante respecto de las decisiones que los Estados estaban realizando respecto de los litigios diplomáticos ${ }^{6}$. Bolivia proporcionó sistemáticamente, a través del enganche, mano de obra para las faenas del nitrato en Tarapacá y Antofagasta.
Esta institución consistía en la contratación de los trabajadores en sus lugares de origen, desde donde eran transportados a las oficinas salitreras de destino bajo la responsabilidad de un representante de las Compañías. Dichos contratos eran generalmente simples acuerdos de palabra que se formalizaban en las oficinas salitreras a través de una libreta de trabajo con un reglamento básico, generalmente con arreglo a los intereses de la empresa.

No solamente la mano de obra fue transfronteriza, sino también el comercio. Desde Bolivia llegaba el ganado ovino y una gran variedad de otros productos como harinas, tejidos, zapatos, cueros, charqui, etc. Conocido fue también el arrieraje argentino que proveyó de mulares para el trabajo y ganado bovino para el consumo alimenticio en las salitreras. Además de los productos derivados del cuero. Esta complementariedad económica que se generó a partir de la economía del nitrato, donde la industria estaba ubicada en el desierto, pero había un espacio de influencia (espacio más que territorio porque se refiere a un mercado) que iba más allá de las fronteras interiores de Chile y de las fronteras internacionales, donde el océano Pacífico, por un lado, y la cordillera de los Andes, por otro, fueron puentes fundamentales para la internacionalización de esa economía minera chilena. Bolivia fue pieza clave en ese espacio de influencia.

Bolivia y Chile durante el ciclo de expansión del salitre dinamizaron sus relaciones comerciales a pesar que, concluida la Guerra del Pacífico, sólo tenían un pacto de tregua. Ambos países coincidieron en un criterio común de desarrollo complementario, que fue la base del acuerdo de 1904. Luis Maira sintetiza muy bien esta coincidencia:

Esto coincidió con la configuración de una fuerte postura en La Paz que concordaba con las demandas chilenas. El presidente Ismael Montes, quien gobernó en dos periodos (1904-1909 y 1913-1918), propuso concentrarse en la extensa superficie que Bolivia todavía conservaba y obtener facilidades para las comunicaciones expeditas hacia los océanos Pacífico y Atlántico. Esto significaba dar prioridad a la construcción del ferrocarril entre Arica y La Paz y al desarrollo de proyectos viales que mejoraran sus nexos con los puertos peruanos y chilenos. Montes aceptó intercambiar el litoral por el financiamiento de estas conexiones, convencido de que la escasez 
de los recursos fiscales bolivianos no permitiría emprender dichas obras durante un tiempo largo. Este criterio, muy bien acogido por los representantes chilenos, fue la base del tratado de paz de 1904, que ambos congresos ratificaron en 1905 para su entrada en vigor (Maira 2004:90).

Este acuerdo permitió un gran tráfico comercial y migraciones transfronterizas, que fueron permanentes desde la misma anexión de la provincia a Chile y durante todo el ciclo de expansión como lo demuestra Luis Castro:

El 6 de diciembre de 1882 el Subdelegado de Pozo Almonte Luis Cevallos le señaló al Jefe Político de la provincia, Patricio Lynch, que el Inspector asentado en la localidad de La Tirana le había hecho saber que por su jurisdicción se internaban comerciantes desde el altiplano y desde Bolivia trayendo distintos productos para comerciarlos en los pueblos salitreros. Su preocupación, sin embargo, no estuvo centrada en la circulación de estos comerciantes y su mercancía, sino en el desconocimiento que él tenía de los derechos aduaneros que se debían cobrar. Tres años más tarde el Subdelegado de Tarapacá, Ismael Aguilar, vuelve a reiterar la misma preocupación (Castro 2005:15).

Como vemos, desde muy temprano la administración chilena observó la presencia de comerciantes bolivianos en las salitreras de Tarapacá, pero no la controla a través de aduanas; en cambio, Bolivia sí lo hizo a través de una Aduanilla en Oruro, como se puede observar en el siguiente documento:

REPUBLICA DE BOLIVIA

OFICINA NACIONAL DE ESTADISTICA

Aduana de: Oruro

Boletín de entradas en el mes de Febrero de 1903

\begin{tabular}{lrllrr}
\hline Por Importación & Bs. & Cs. & Por Exportación & Bs. & Cs. \\
\hline Ultramar & 1032.4 & Barrilla de Estaño & 20.000 & 4 \\
Manifiesto & 199 & Pastas y M. de Plata & 1.055 & 11 \\
Almacenaje & 836.3 & Goma Elástica & 57 & 40 \\
Derechos Dobles & 58.8 & & & \\
Encomiendas Postales & 31.35 & & & \\
& 2157.85 & & 21.112 & 55 \\
\hline
\end{tabular}

(Archivo de la Prefectura de La Paz, Documentos sobre el litoral).
La economía del salitre no solamente tuvo un impacto en la mano de obra que iba y venía hacia y desde las salitreras, sino también en los bienes y productos que llegaban desde ultramar, generando un cambio de hábitos no sólo en Tarapacá sino también en Bolivia. Se sabe del comercio de cocinas y planchas de hierro, zinc (calaminas) para los techos, leche condensada, ropa y telas, muebles, etc. Posiblemente, también se internaron libros y revistas llegados desde Europa.

\section{La población boliviana en las oficinas salitreras de Tarapacá}

Los flujos de trabajadores bolivianos (y sus familias) que se dirigían a las salitreras de Tarapacá cruzando la frontera lo hacían preferentemente por Pisiga, bajaban por los valles de Camiña, Aroma y Tarapacá, destacándose la presencia cochabambina (González 1995). La población boliviana estaba en toda la provincia, pero era más notoria en los cantones del norte, entre Zapiga y Huara. En cambio, en los cantones de Antofagasta y de Taltal la población peruana y boliviana tuvo una escasa presencia. En la Tabla 1, construida a partir de un libro de salitreras en propiedad del autor, se puede observar la distribución de trabajadores en el mes de abril de 1904, un mes que no está afectado por el movimiento poblacional por razones agrícolas o de otro tipo, y un año de auge y relativamente estable (además el año del Tratado de Paz y Amistad entre Bolivia y Chile).

Según la Estadística Oficial de 1902, en la industria salitrera se ocuparon 24.538 hombres, distribuyéndose las nacionalidades de la siguiente forma: 17.461 chilenos (71\%), 3.352 bolivianos (14\%) y 2.805 peruanos (11\%). En cambio, el censo oficial de 1907 registró 82.126 chilenos (49,7\%), 23.574 peruanos $(14,2 \%)$ y 15.652 bolivianos $(9,5 \%)$ y de un total de 165.302 habitantes en la provincia. No es de extrañar que la presencia boliviana sea mayor que la peruana entre los trabajadores de las salitreras. Los bolivianos llegaban a trabajar estacionalmente a las salitreras, incluso con sus familias, para regresar a Bolivia una vez concluido un periodo, sin haber tenido la necesidad de bajar a los puertos o caletas del litoral. La población peruana, en cambio, estaba también radicada en los puertos de Iquique y Pisagua, y en pueblos precordilleranos como Camiña, Tarapacá, Pica, Huatacondo, entre otros. 
Tabla 1. Operarios chilenos, peruanos y bolivianos en las salitreras, abril de 1904.

Chilean, Peruvian and Bolivian operators in the saltpeter deposits, April, 1904.

\begin{tabular}{lcccccccccc}
\hline \multirow{2}{*}{ Salitreras } & \multicolumn{2}{c}{ Chilenos } & \multicolumn{2}{c}{ Peruanos } & \multicolumn{2}{c}{ Bolivianos } & \multicolumn{2}{c}{ Otras } & \multicolumn{2}{c}{ Totales } \\
\cline { 2 - 11 } & $\mathrm{N}^{\circ}$ & $\%$ & $\mathrm{~N}^{\circ}$ & $\%$ & $\mathrm{~N}^{\circ}$ & $\%$ & $\mathrm{~N}^{\circ}$ & $\%$ & $\mathrm{~N}^{\circ}$ & $\%$ \\
\hline San Antonio de Zapiga & 210 & 67,7 & 70 & 22,6 & 25 & 8,1 & 5 & 1,6 & 310 & 100,0 \\
San Patricio, Dolores & 31 & 13,2 & 33 & 14,1 & 170 & 72,6 & 0 & 0,0 & 234 & 100,0 \\
Ángela, Santa Catalina & 19 & 5,4 & 12 & 3,4 & 320 & 90,7 & 2 & 0,6 & 353 & 100,0 \\
Puntunchara, Negreiros & 211 & 63,9 & 75 & 22,7 & 37 & 11,2 & 7 & 2,1 & 330 & 100,0 \\
Primitiva, Negreiros-Huara & 145 & 39,2 & 65 & 17,6 & 98 & 26,5 & 62 & 16,8 & 370 & 100,0 \\
Antofagasta, Cantón Central & 494 & 94,8 & 7 & 1,3 & 8 & 1,5 & 12 & 2,3 & 521 & 100,0 \\
Alemania, Taltal & 425 & 94,2 & 7 & 1,6 & 4 & 0,9 & 15 & 3,3 & 451 & 100,0 \\
\hline
\end{tabular}

Fuente: Libro de registro de la Asociación Salitrera de Propaganda.

Estas cifras no dejan dudas que a partir del siglo XX la pampa salitrera, a nivel obrero, fue una sociedad compuesta preferentemente por tres nacionalidades: chilena, boliviana y peruana, en ese orden de importancia poblacional. En todas las salitreras chilenos, bolivianos y peruanos convivieron estrechamente en los campamentos, con sus diferencias y sus encuentros, pero ello fue particularmente evidente en la provincia de Tarapacá; porque si bien en Antofagasta se dio un fenómeno similar fue menos significativo proporcionalmente hablando, donde la población boliviana tuvo efectivamente una presencia muy relevante pero la peruana fue menos significativa (lo que se puede observar en la Tabla 2). En Taltal, en tanto, prácticamente no existió este fenómeno socio-cultural. La importancia de la población boliviana en Tarapacá y Antofagasta se explica de modo fundamental por la explotación del nitrato, aunque no de modo exclusivo, porque hubo otras fuentes de atracción de mano de obra boliviana como Caracoles en Antofagasta y Collahuasi en Tarapacá.

La vida cotidiana unió en Tarapacá a peruanos, bolivianos y chilenos en las cantinas, las calicheras, los clubes, las filarmónicas, los gremios, las cofradías y en los movimientos sociales, como las huelgas. Una de las fuentes documentales más importantes sobre la presencia boliviana en las salitreras de Tarapacá son los informes del Cónsul de Bolivia en Iquique, don Arístides Moreno, a la Cancillería de su país. Veamos un fragmento de uno de esos informes, correspondiente al año 1907:
(...) Pasando a la Provincia en general y á la población boliviana en particular, debo suministrar los datos siguientes:

En 1895 tenía 89.751 habitantes toda la Provincia y en el Censo tomado doce años después (28 de noviembre de 1907) resultó con la población que sigue:

69.262 Chilenos

23.574 Peruanos

12.528 Bolivianos

7.672 de Otras nacionalidades

110.036 habitantes

He aquí algunos detalles respecto de sexos y residencias de los pobladores bolivianos á que acabo de referirnos:

Población Urbana

7.268 hombres y 3.802 mujeres ó sea 11.070 Id Rural

735 “ 723 “ “ 1.458

TOTALES

8.003 hombres 4.525 mujeres “ 12.528

Residiendo 6.700 en el Departamento de Tarapacá y 5.828 en el de Pisagua, o sea 12.528 en toda la Provincia. La gran mayoría de la población boliviana que vive en los minerales de Collahuasi, es de las departamentos de Oruro y Potosí (de 
Tabla 2. Extranjeros en las provincias de Tarapacá y Antofagasta, 1907.

Foreigners in the Provinces of Tarapacá and Antofagasta, 1907.

\begin{tabular}{|c|c|c|c|c|c|c|}
\hline \multirow{2}{*}{ Nacionalidad } & \multicolumn{3}{|c|}{ Tarapacá } & \multicolumn{3}{|c|}{ Antofagasta } \\
\hline & Hombres & Mujeres & Total & Hombres & Mujeres & Total \\
\hline Bolivia & 8.003 & 4.525 & 12.528 & 3.555 & 2.273 & 5.828 \\
\hline Perú & 13.928 & 9.646 & 23.574 & 1.194 & 555 & 1.749 \\
\hline África & 0 & 0 & 0 & 3 & 1 & 4 \\
\hline Alemania & 629 & 64 & 693 & 111 & 67 & 1.178 \\
\hline Argentina & 409 & 147 & 556 & 1.410 & 469 & 1.879 \\
\hline Australia & 3 & 0 & 3 & 0 & 0 & 0 \\
\hline Austria Hung & 366 & 49 & 415 & 766 & 124 & 890 \\
\hline Balkanes & 10 & 0 & 10 & 5 & 0 & 5 \\
\hline Bélgica & 13 & 3 & 16 & 20 & 0 & 20 \\
\hline Brasil & 11 & 4 & 15 & 20 & 2 & 22 \\
\hline Canadá & 8 & 1 & 9 & 9 & 0 & 9 \\
\hline Centro América & 11 & 2 & 13 & 9 & 0 & 10 \\
\hline Colombia & 33 & 7 & 40 & 29 & 1 & 30 \\
\hline China & 1.329 & 6 & 1.335 & 287 & 0 & 287 \\
\hline Dinamarca & 43 & 2 & 45 & 57 & 4 & 61 \\
\hline Ecuador & 107 & 37 & 144 & 65 & 7 & 72 \\
\hline Egipto & 2 & 0 & 2 & 2 & 1 & 3 \\
\hline España & 686 & 131 & 817 & 1.049 & 149 & 1.198 \\
\hline Estados Unidos & 141 & 19 & 160 & 224 & 3 & 227 \\
\hline Francia & 381 & 52 & 433 & 282 & 46 & 328 \\
\hline Gran Bretaña & 1.278 & 117 & 1.395 & 1.929 & 122 & 2.051 \\
\hline Grecia & 40 & 0 & 40 & 116 & 0 & 116 \\
\hline Holanda & 43 & 7 & 50 & 66 & 0 & 66 \\
\hline Italia & 823 & 203 & 1.026 & 864 & 104 & 968 \\
\hline Japón & 95 & 3 & 98 & 55 & 0 & 55 \\
\hline Mejico & 31 & 3 & 34 & 20 & 1 & 21 \\
\hline Noruega & 46 & 1 & 47 & 138 & 0 & 138 \\
\hline Paraguay & 2 & 0 & 2 & 5 & 0 & 5 \\
\hline Portugal & 34 & 1 & 35 & 26 & 4 & 30 \\
\hline Rusia & 27 & 1 & 28 & 107 & 8 & 115 \\
\hline Suecia & 67 & 2 & 69 & 85 & 1 & 86 \\
\hline Suiza & 19 & 1 & 20 & 25 & 4 & 29 \\
\hline Turquia & 81 & 9 & 90 & 237 & 42 & 279 \\
\hline Uruguay & 12 & 6 & 18 & 27 & 6 & 33 \\
\hline Venezuela & 12 & 2 & 14 & 8 & 0 & 8 \\
\hline Totales & 28.723 & 15.051 & 43.774 & 13.805 & 3.995 & 17.800 \\
\hline
\end{tabular}

Fuente: Censo de 1907 (en Silva 1909). 
este último sobre todo) y en el resto de la Provincia predominan, por su número los cochabambinos. (Archivo Intendencia de Tarapacá, Consulados, 1907).

Este informe señala la distribución de los bolivianos en la provincia de Tarapacá, destacando la presencia cochabambina, la que aparte de ser notoriamente más numerosa que las de otras regiones de ese país, se concentraba en las salitreras, a diferencia de los paceños y orureños que prefirieron las minas de cobre y plata.

La población boliviana en Tarapacá se resistió a abandonar la provincia, a pesar de la gran crisis de 1930. Pero, su partida no fue diferente de la población peruana y chilena, pues el desierto comenzó a despoblarse porque ya no había puestos de trabajo. Sin embargo, ella dejó una huella imborrable en la sociedad del norte chileno, donde sus tradiciones y costumbres todavía pueden observarse no sólo entre sus descendientes, sino en toda la sociedad.

\section{Conclusiones}

La frontera de las provincias ocupadas por Chile, después de la Guerra del Pacífico, con Perú y Bolivia, debió construirse durante décadas de negociaciones diplomáticas, pero también fue producto del uso y la dinámica transfronteriza, donde el enclave salitrero tuvo un papel importante como catalizador de población que terminó identificándose con Chile.

Específicamente, la frontera de Tarapacá con Bolivia, que podría suponerse ya consolidada antes de 1879 entre los estados peruano y boliviano, debió también ser estudiada por los peritos para ser definida en forma definitiva en el marco del Tratado de 1904, donde los comuneros alto andinos tuvieron un papel significativo no sólo como informantes, sino también como protagonistas en la construcción de dicha frontera.

La explotación del salitre fue desde el comienzo intensiva en mano de obra y requirió de flujos de trabajadores provenientes de las regiones circunvecinas a Tarapacá y Antofagasta, donde el "enganche" fue la institución más conocida. De tal modo, el enclave salitrero necesitó de fronteras abiertas que posibilitaran una circulación expedita de mano de obra para las faenas de extracción y elaboración del nitrato. Entre el término de la guerra y la crisis del salitre (1883 y 1930) las fronteras no tuvieron un tratamiento homogéneo. La frontera con Perú no estaba definida y, por lo mismo, fue de conflicto; en cambio, la frontera con Bolivia fue de integración económica, donde los circuitos comerciales fueron la prioridad para las relaciones diplomáticas.

Durante el ciclo de expansión del salitre 18801930 toda la región salitrera, desde Pisagua a Taltal pero especialmente la provincia de Tarapacá, vivió un fenómeno cultural y sociológico diferente al resto del país, pues recibió miles de trabajadores venidos desde los cuatro puntos cardinales, en especial de Bolivia, allende los Andes.

Esta migración transfronteriza fue posibilitada por una política internacional chilena y otra boliviana pragmáticas, que privilegiaron acuerdos que beneficiaran las actividades mineras en ambos países. Los obreros bolivianos llegaron a trabajar a las salitreras de Tarapacá, primero bajo el amparo de un Pacto de Tregua y luego de un Tratado de Paz y Amistad, pero sobre todo lo hicieron bajo el amparo de los propios empresarios del salitre que requerían mano de obra para las faenas de extracción y elaboración del nitrato. De esta manera se generó una movilidad transfronteriza compleja, tanto por los flujos de bienes y personas como por las dimensiones culturales de la misma. La importancia de esta migración fue tal que, unido al origen peruano de esta provincia, se puede decir que durante el período salitrero, desde un punto de vista demográfico, Tarapacá fue un territorio sociológicamente tri-nacional: peruano, boliviano y chileno, pero bajo un estado de derecho y soberanía chilenos y una economía salitrera internacional (Blakemore 1977).

La presencia boliviana durante el ciclo de expansión del salitre no sólo dejó una marca en la cultura nortina, sino también quedaron importantes obras de ingeniería, como los ferrocarriles y caminos que todavía siguen consolidándose entre Tarapacá y Bolivia.

La explotación del salitre posibilitó que entre Chile y Bolivia existiese un proceso de integración física y poblacional sólo comparable a la atracción que ejerció la mina de plata de Potosí durante la Colonia; por ello, consideramos que este capítulo de las relaciones del norte de Chile con las regiones bolivianas territorialmente contiguas, e incluso algunas no contiguas como Cochabamba, amerita una investigación multidisciplinaria que sintonice con la importancia que merece.

La presencia de trabajadores bolivianos (y también peruanos) en la historia del nitrato 
merece un capítulo especial, incluso en la historia del movimiento obrero salitrero. Son escasas las referencias bibliográficas y no alcanzan a dimensionar la verdadera importancia de esta población en tierras del salitre, donde llegaron a conformar con chilenos y otros grupos humanos como chinos cantoneses, croatas, italianos, españoles, argentinos, británicos, alemanes, etc., una sociedad multicultural.

Agradecimientos: Este trabajo es resultado parcial del Proyecto Fondecyt $\mathrm{N}^{\circ} 1070032$ y del Proyecto ECOS/CONICYT N ${ }^{\circ} 404 \mathrm{H} 03$. Se agradecen los comentarios de los evaluadores de la revista.

\section{Referencias Citadas}

Blakemore, $\mathrm{H}$.

1977 Gobierno Chileno y Salitre Inglés 1886-1896. Balmaceda y North. Editorial Andrés Bello, Santiago.

1996 Historia del Ferrocarril de Antofagasta a Bolivia, 1888-1988. Edición Mercedes Gajú, Santiago.

Castro, L.

1995 Tarapacá 1880-1936: debates, reflexiones, propuestas y proyectos en torno al problema del desarrollo. Temas Regionales 2 (1):32-66.

2005 Tráfico mercantil, comerciantes indígenas y fiscalización estatal durante el Ciclo Salitrero (Tarapacá 1880-1930). Manuscrito en posesión del autor.

2008 El Estado chileno, los agentes fiscales y el temprano ordenamiento administrativo del espacio andino de la provincia de Tarapacá (1880-1930). Chungara Revista de Antropología Chilena 40:219-233.

González, S.

1995 Cochabambinos de habla quechua en las salitreras de Tarapacá (1880-1930). Chungara: 27:135-151.
2006 El Toco y la guerra del Pacífico: una hipótesis de trabajo. Hombre y Desierto: Una Perspectiva Cultural 13:7-28. Maira, L.

2004 Las relaciones entre Chile y Bolivia en el centenario del tratado de 1904. Foreign Affairs en Español 4 (4):89-103.

Palacios, R.

1974 La Chilenización de Tacna y Arica 1883-1929. Colección Perú Historia, Editorial Jurídica S.A., Lima.

Riso Patrón, L.

1910 La Línea de la Frontera con la República de Bolivia. Sociedad Imprenta y Litografía Universo, Santiago. Silva, D.

1909 Guía Administrativa, Industrial y Comercial de Tacna, Tarapacá y Antofagasta. Imprenta Gutenberg, Santiago.

\section{Fuentes}

Archivo Intendencia de Tarapacá: Consulados, 1907.

Archivo Intendencia de Tarapacá: Subdelegaciones, libro 7, 1910.

Prefectura de La Paz. Documentos sobre el Litoral.

\section{Notas}

1 Fue ratificado por el Congreso peruano el 28 de marzo del año siguiente.

2 Sistema de contratación de mano de obra utilizado por las compañías salitreras a través de encargados o "enganchadores".

3 El caso de Chilcaya fue discutido en la disputa diplomática entre Perú y Chile en el marco del plebiscito por Tacna y Arica, pues los diplomáticos peruanos señalaban que Chilcaya (y sus borateras) pertenecía a la provincia de Arica y los chilenos a Tarapacá.

$4 \quad$ La evolución de la población venida desde los países limítrofes hacia el territorio salitrero tuvo un comportamiento bien definido de crecimiento y decadencia que se correlaciona con el desarrollo del ciclo de expansión del nitrato a partir de 1880, que es generado tanto por la introducción de nuevas tecnologías en la lixiviación del salitre (sistema Shanks), debido a la nueva política estatal bajo la administración chilena, como por la introducción de capitales a través de sociedades anónimas con la creación de nuevas compañías salitreras, entre otros factores.

5 Como lo enseña la siguiente documentación: COPIA: SELLO DE LA SUBPREFECTURA.- $\mathrm{N}^{\circ}$ 408.- SAN CRISTÓBAL, OCTUBRE 18 DE 1910.- AL SEÑOR SUBDELEGADO DE TARAPACÁ, CHILE.- SEÑOR.- CONFIRMÁNDOLE MI ANTERIOR OFICIO N ${ }^{\circ} 386$, FECHADO EN 21 DE AGOSTO ÚlTIMO, QUE DIRIJÍO A UD., CON MOTIVO DE QUE LOS VECINOS NATURALES DE
ESA REPÚBLICA, RESIDENTES EN EL LUGAR DENOMINADO CARIQUIMA, COMPRENSIÓN DE ESE DISTRITO: PASCUAL, TIBURCIO Y MARCELO ESTEVAN, TODOS DEL MISMO APELLIDO, HAN COMETIDO HECHOS QUE CONSTITUYEN AVANCES DE TERRITORIO BOLIVIANO, HABIENDO VERIFICADO TRABAJOS DE BARBECHO EN LA LÍNEA DIVISORIA DEL CANTÓN LLICA, COMO LE TENGO COMUNICADO A UD., Y DESTRUYENDO EN ESPECIAL LOS LINDEROS LIMÍTROFES MARCADOS CON LOS $\mathrm{N}^{\circ}$ 36 AL 55 EN LOS LUGARES APACHETA DE TOLA, Y ALTO DE PAHUATALLA; CON CUYO MOTIVO SE HA RECIBIDO EN ESTA SUBPREFECTURA, ORDENES IMPARTIDAS POR MI GOBIERNO, ASÍ COMOTAMBIÉN POR PARTE DEL SEÑOR PREFECTOY COMANDANTE GENERAL DE ESTE DEPARTAMENTO (POTOSÍ) AFECTO DE QUE EN LO SUCESIVO SE EVITEN IGUALES AVANCES SOBRE TERRITORIO BOLIVIANO. EN VIRTUD A ESTAS PREVENCIONES, INSINÚO A SU AUTORIDAD SE SIRVA ORDENAR A QUIEN CORRESPONDA LA CONVENIENCIA DE QUE RESTABLEZCA LOS MOJONES DERRIBADOS, POR HABER SIDO COLOCADO ESTOS LINDEROS DE ACUERDO ENTRE LOS COMISIONADOS DE LÍMITES DE AMBAS NACIONES.- ESPERANDO OBTENER RESPUESTA FAVORABLE SOBRE EL PARTICULAR, REITERO A UD MIS CONSIDERACIONES DE ESTIMA, CON QUE 
ME SUSCRIBO ATENTO.- SERVIDOR.- FIRMADO: Y DEL CASTILLO. COPIA DE LA CONTESTACIÓN.SELLO DE LA SUBDELEGACIÓN.- TARAPACÁ 5 DE NOVIEMBRE DE 1910.- No 93.- SEÑOR SUBPREFECTO DE NOR LIPEZ.- SAN CRISTÓBAL- BOLIVIA. SEÑOR.EN MI PODER SU ATENTA FECHA 18 DE OCTUBRE QUE ES LA PRIMERA Y ÚNICA QUE HA RECIBIDO SOBRE ATROPELLOS Y ÚNICA QUE HE RECIBIDO SOBRE ATROPELLOS Y AVANCE EN EL TERRITORIO BOLIVIANO COMETIDO POR PASCUAL, TIBURCIOY MARCELO ESTEBAN.- EN CONTESTACIÓN LE DIRÉ
QUE HOY MISMO DARÉ PARTE DE ELLA, AL SEÑOR INTENDENTE DE LA PROVINCIA, Y PEDIRÉ INFORME AL INSPECTOR DE CARIQUIMA, Y ME PERMITO ASEGURAR A UD QUE SI ESOS HECHOS SON CIERTOS NO SE VOLVERÁN A REPETIR.- DIOS GUARDE A UD., FIRMA MAURICIO REYNAUD.- SUBDELEGADO (Archivo Intendencia de Tarapacá, Subdelegaciones).

6 A diferencia de la población de las provincias de Tacna y Arica que fueron consideradas para participar en un plebiscito respecto de la soberanía de esos territorios, de parte Perú o Chile. 
